\title{
ON PRODUCT EXPANSIONS OF THETA-FUNCTIONS
}

\author{
A. P. OGG
}

In [1], Hecke set up a general method of passing back and forth between Dirichlet series and functions on the upper half plane (via the Mellin transform) and showed how functional equations on the one side correspond to functional equations on the other. Weil [2] has remarked that this method is applicable in a more general setting than in [1], and has shown in this manner that the functional equation for Dedekind's function $\eta(\tau)$ is an immediate consequence of the functional equation for the Riemann zeta-function. In this note we give a proof along these lines of the product expansion of the basic theta-function $\vartheta=\vartheta_{3}=\vartheta_{\infty}$.

We start with

$$
\vartheta(\tau, x)=\sum_{n} e^{2 \pi i n x+i \pi n \tau}
$$

where $x, \tau \in C, \operatorname{Im}(\tau)>0$ (the sum being over $n \in Z$ ), and its transformation formula

$$
e^{x i \tau x^{2} \vartheta(\tau, \tau x)=(\tau / i)^{-1 / 2} \vartheta(-1 / \tau, x),}
$$

which follows, for example, from expressing the left side $\sum_{n} e^{x i r(n+x)^{2}}$ as a Fourier series in $x$. We set

$$
\begin{aligned}
f_{1}(\tau, x) & =e^{\pi i \tau x^{2} \vartheta(\tau, \tau x),} \\
f_{2}(\tau, x) & =\vartheta(\tau, x), \\
A_{j}(x) & =\lim _{\operatorname{Im} \tau_{\rightarrow \infty}} f_{j}(\tau, x),
\end{aligned}
$$

so $A_{2}(x)=1$ and $A_{1}(x)=0$ if $x \notin Z, A_{1}(x)=1$ if $x \in Z$. We can rewrite (2) as

$$
f_{j}(-1 / \tau, x)=(\tau / i)^{1 / 2} f_{k}(\tau, x),
$$

where $\{j, k\}=\{1,2\}$. Now

$$
\pi^{-s} \Gamma(s) x^{-s}=\int_{0}^{\infty} t^{-1} e^{-x x t} d t, \quad(\operatorname{Re} s>0)
$$

for $x>0$, so for $x \in R$ we have

Received by the editors June 17, 1968. 


$$
\begin{aligned}
\Phi_{j}(2 s, x) & =\pi^{-s} \Gamma(s) \phi_{j}(2 s, x) \\
& =\int_{0}^{\infty} t^{-1}\left(f_{j}(i t, x)-A_{j}(x)\right) d t \quad(\operatorname{Re} s>1)
\end{aligned}
$$

where

$$
\phi_{1}(s, x)=\sum_{n}(n+x)^{-\triangleleft}
$$

a general Dirichlet series, and

$$
\phi_{2}(s, x)=\sum_{n=1} 2 \cos (2 \pi n x) n^{-s} .
$$

In (7) we have adopted the (nonstandard) conventions that $0^{-s}=0$ and $x^{-s}=|x|^{-s}=e^{-s \log |x|}$. Note that $\phi_{j}(s, x)=2 \zeta(s)$ if $x \in Z$, and that

$$
\phi_{2}(2, x+1 / 2)=2 \pi^{2}\left(x^{2}-1 / 12\right) \quad \text { for }|x| \leqq 1 / 2
$$

as follows from computing the Fourier series of the right side on the interval $[-1 / 2,1 / 2]$.

Theorem 1. For $\{j, k\}=\{1,2\}, \Phi_{j}(s, x)+2 A_{j}(x) / s+2 A_{k}(x) /(1-s)$ is entire, bounded in every vertical strip, and satisfies $\Phi_{j}(s, x)=$ $\Phi_{k}(1-s, x)$.

We omit the proof, which is standard: (Cf. [1], Hauptsatz.)

Now let $\vartheta_{0}(\tau, x)$ be defined by the product

$$
\vartheta_{0}(\tau, x)=\prod_{n=1}\left(1-q^{2 n}\right)\left(1+z q^{2 n-1}\right)\left(1+z^{-1} q^{2 n-1}\right)
$$

where $q=e^{\pi i r}, z=e^{2 \pi i x}$; we will show eventually that $\vartheta=\vartheta_{0}$. Note that $\vartheta_{0}(\tau, x)$ has zeroes only at $x \equiv(\tau+1) / 2$ modulo 1 and $\tau$. Consider

$$
\begin{aligned}
g(\tau, x) & =\log \vartheta_{0}(\tau, x) \\
& =\sum_{n=1} \sum_{m=1}\left(-q^{2 m n} / m+(-1)^{m-1} q^{m(2 n-1)}\left(z^{m}+z^{-m}\right) / m\right) .
\end{aligned}
$$

The inverse Mellin transform

$$
e^{\pi i r y}=\frac{1}{2 \pi i} \int_{0-c}(\tau / i)^{-s} \Gamma(s)(\pi y)^{-\triangleleft} d s
$$

(where $y>0, \sigma=\operatorname{Re}(s)$, and $c>0$ ) gives, for real $x$,

$$
g(\tau, x)=\frac{1}{2 \pi i} \int_{\sigma=c}(\tau / i)^{-8} \Psi_{1}(s, x) d s,
$$


$(c>1)$, where

$$
\begin{aligned}
& \Psi_{1}(s, x)=(2 \pi)^{-s} \Gamma(s) \psi_{1}(s, x) \\
& \psi_{1}(s, x)=\zeta(s)\left(-\zeta(s+1)+\left(1-2^{s}\right) \phi_{2}(s+1, x+1 / 2)\right) .
\end{aligned}
$$

Now Theorem 1 (including the functional equation of the zetafunction) and the formula

$$
\pi^{1 / 2} \Gamma(s)=2^{o-1} \Gamma(s / 2) \Gamma((s+1) / 2),
$$

give immediately

$$
\Psi_{1}(-s, x)=\Psi_{2}(s, x)
$$

where

$$
\begin{aligned}
& \Psi_{2}(s, x)=(2 \pi)^{-s} \Gamma(s) \psi_{2}(s, x), \\
& \psi_{2}(s, x)=\zeta(s+1)\left(-\zeta(s)+\left(1-2^{-s}\right) \phi_{1}(s, x+1 / 2)\right) .
\end{aligned}
$$

Now $\left(1-2^{-s}\right) \zeta(s+1)=\sum_{m=1}(-1)^{m-1} m^{-s} / m$, and $2^{-s} \phi_{1}(s, x+1 / 2)$ $=\sum_{n=1}\left\{(2 n-1+2 x)^{-s}+(2 n-1-2 x)^{-s}\right\}$. Hence

$$
\begin{aligned}
& \frac{1}{2 \pi i} \int_{\sigma=c}(\tau / i)^{-\triangleleft} \Psi_{2}(s, x)=g(\tau, \tau x) \\
& \quad=\sum_{n, m=1}\left(-q^{2 m n} / m+(-1)^{m-1}\left(e^{\pi i \tau(2 n-1+2 x) m}+e^{\pi i \tau(2 n-1-2 x) m}\right) / m\right) .
\end{aligned}
$$

Now assume $-1 / 2 \leqq x \leqq 1 / 2$. By (13)-(17), $\Psi_{1}(s, x)$ is regular except possibly for poles at $s=0, \pm 1$. By Theorem 1, $\left(1-2^{s}\right) \phi_{2}(s+1, x+1 / 2)$ vanishes at $s=0$, so $\psi_{1}(s, x)+\zeta(s) \zeta(s+1)$ is regular at $s=0$, whence $\Psi_{1}(s, x)-\left(2 s^{2}\right)^{-1}$ is regular at $s=0$, using the fact that $\zeta(0)=-1 / 2 . \phi_{1}(s, x+1 / 2)$ has a simple pole of residue 2 at $s=1$, so $\Psi_{2}(s, x)$ is regular at $s=1$ and hence $\Psi_{1}(s, x)$ is regular at $s=-1$. Finally, $\Psi_{1}(s, x)$ has a simple pole of residue $-\pi x^{2}$ at $s=1$, by (9). Moving the line of integration to the left, computing the residues, and applying (15), we get

$$
\begin{aligned}
g(-1 / \tau, x) & =\frac{1}{2 \pi i} \int_{\sigma=c>1}(\tau / i)^{\curvearrowright} \Psi_{1}(s, x) d s \\
& =\pi i x^{2} \tau+1 / 2 \log (\tau / i)+\frac{1}{2 \pi i} \int_{\sigma=c<-1}(\tau / i)^{\curvearrowright} \Psi_{1}(s, x) d s \\
& =\pi i x^{2} \tau+1 / 2 \log (\tau / i)+\frac{1}{2 \pi i} \int_{\sigma=c>1}(\tau / i)^{-\diamond} \Psi_{2}(s, x) d s \\
& =\pi i x^{2} \tau+1 / 2 \log (\tau / i)+g(\tau, \tau x) .
\end{aligned}
$$


Exponentiating, we have

$$
\vartheta_{0}(-1 / \tau, x)=(\tau / i)^{1 / 2} e^{\pi i x^{2}} \vartheta_{0}(\tau, \tau x)
$$

(now for all $x \in C$ ), the same functional equation (2) as for $\vartheta(\tau, x)$. It follows that the quotient $\vartheta(\tau, x) / \vartheta_{0}(\tau, x)$ is an elliptic function of $x$ with periods 1 and $\tau$, and at most one simple pole (modulo periods) at $x=(\tau+1) / 2$; this elliptic function is therefore constant, hence $\vartheta(\tau, 0) / \vartheta_{0}(\tau, 0)$ is constant. Finally, $\vartheta_{0}(\tau, 0)$ is determined as a function of $\tau$ by its functional properties (cf. [1, Satz 2a]), so is a constant times $\vartheta(\tau, 0)$; letting $\operatorname{Im}(\tau)$ tend to $\infty$, we see the constant is 1 . Thus:

TheOREM 2. $\vartheta(\tau, x)$ has the product expansion

$$
\prod_{n=1}^{\infty}\left(1-q^{2 n}\right)\left(1+z q^{2 n-1}\right)\left(1+z^{-1} q^{2 n-1}\right),
$$

where $q=e^{\pi i \tau}, z=e^{2 \pi i x}$.

\section{REFERENCES}

1. E. Hecke, Über die Bestimmung Dirichletscher Reihen durch ihre Funktionalgleichung, Math. Ann. 112 (1936), 664-699.

2. A. Weil, Sur une formule classique, J. Math. Soc. Japan 20 (1968), 400-402.

University of California AT BERKELEY 